
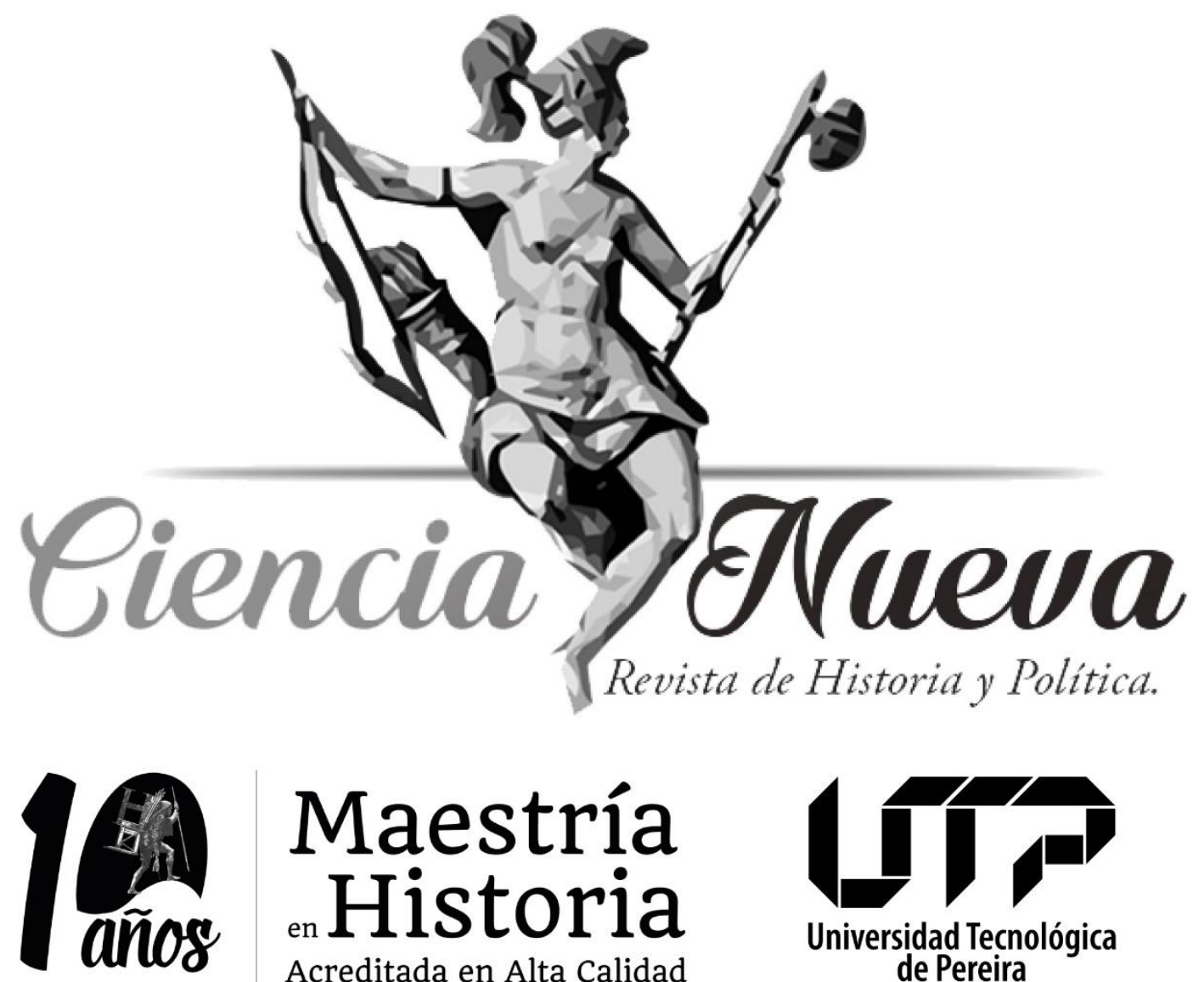

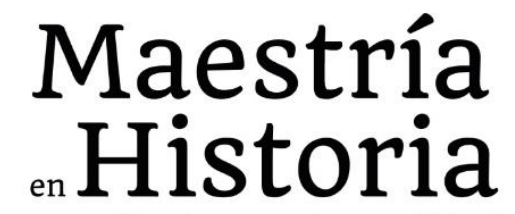

Acreditada en Alta Calidad

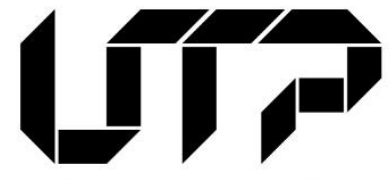

Universidad Tecnológica de Pereira

RESEÑAS

CORREA RAMÍREZ, JHON JAIME, ANDERSON PAUL GIL PÉREZ Y NATALIA AGUDELO CASTAÑEDA. RESIGNIFICANDO LA HISTORIA DE LA UNIVERSIDAD TECNOLÓGICA DE PEREIRA. AJUTP: MEMORIAS QUE NO SE JUBILAN. PEREIRA: EDITORIAL UNIVERSIDAD TECNOLÓGICA DE PEREIRA, 2020

CORREA RAMÍREZ, JHON JAIME, ANDERSON PAUL GIL PÉREZ Y NATALIA AGUDELO CASTAÑEDA. RESIGNIFYING THE UNIVERSIDAD TECNOLÓGICA DE PEREIRA'S HISTORY. AJUTP: MEMORIES WHICH DON'T RETIRE. PEREIRA: EDITORIAL UNIVERSIDAD TECNOLÓGICA DE PEREIRA, 2020 Michael Stiven Valencia Villa pp. 200-205

DOI: https://doi.org/10.22517/25392662.24485

Vol. 4 Núm. 2 | Julio-diciembre de 2020

Pereira, Colombia

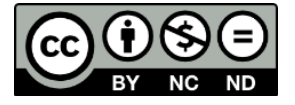




\title{
Correa Ramírez, Jhon Jaime, Anderson Paul Gil Pérez y Natalia Agudelo CASTAÑEDA. RESIGNIFICANDO LA HISTORIA DE LA UNIVERSIDAD TECNOLÓGICA DE Pereira. AJUTP: Memorias QUe no SE JUbilan. Pereira: Editorial UniVersidad TeCnológica de Pereira, 2020*
}

\author{
Correa Ramírez, Jhon Jaime, Anderson Paul Gil Pérez y natalia Agudelo \\ Castañeda. Resignifying the Universidad TecnOlógica de Pereira's history. \\ AJUTP: MEMORIES WHICH DON'T RETIRE. PEREIRA: EDITORIAL UNIVERSIDAD TECNOLÓGICA \\ DE PEREIRA, 2020
}

Michael Stiven Valencia Villa** maies1222@utp.edu.co

ORCID: https://orcid.org/0000-0003-4475-0391

\begin{aligned} & \hline Recibido: 09 de septiembre de 2020. \\ & Revisado: 19 de septiembre de 2020. \\ & Aceptado: 23 de septiembre de 2020. \\ & Publicado: 31 de diciembre de 2020. \\ & \hline\end{aligned}

Parece pretencioso el título que los autores Correa, Gil y Agudelo han definido para la publicación de su trabajo, que es producto de un proceso de investigación de largo aliento con la Asociación de Jubilados de la Universidad Tecnológica de Pereira. Al propósito de resignificar la historia de la institución, en efecto, subyacen varios intereses de gran calado, entre los que se encuentran la superación de la fundación como matriz explicativa del desarrollo de la Universidad, el carácter teleológico que acompaña las revisiones documentales realizadas en función de las proyecciones estratégicas y la reivindicación de un grupo - los jubilados - que reclama participación en la conducción de la alma mater.

El resultado es una obra polifónica que brinda al lector una serie de nuevas interpretaciones sobre un período que va desde el inicio de actividades académicas en 1961 hasta los primeros años de la rectoría del ingeniero Luis Enrique Arango, en la primera década del siglo XXI. Además, se añaden algunas puntadas reflexivas sobre las transformaciones en el ámbito laboral que se observan desde el presente. La obra se concentra en los aspectos cotidianos, las trayectorias y la política universitaria sin pretender convertirse en un nuevo relato hegemónico, sino todo lo contrario: una apertura del horizonte de experiencias de la vida universitaria.

De entrada, la obra plantea un diálogo de ida y vuelta entre memoria e historia, en el que la objetivación de la primera exige apertura e inclusión de la segunda. En tal sentido, conviene entender la memoria como un fenómeno que siempre corresponde al presente; por naturaleza es dialéctica entre el recuerdo y el olvido, permanece viva al estar encarnada en grupos sociales dispuestos a su revitalización y es por tanto dinámica, pero frágil y volátil ${ }^{1}$. Es precisamente a esta última característica a la que buscan responder los historiadores, a

\footnotetext{
* Este documento respeta las directrices y normas dispuestas en la Declaración de Ética de Publicación de Ciencia Nueva, Revista de Historia y Política. Esta declaración puede consultarse en la página web de la revista: http://revistas.utp.edu.co/index.php/historia

* Licenciado en Comunicación en Informática Educativa y estudiante de la Maestría en Historia de la Universidad Tecnológica de Pereira. Integrante del Grupo de Investigación Políticas, Sociabilidades y Representaciones Histórico-Educativas, PSORHE.

${ }^{1}$ Pierre Nora, Los lugares de la memoria (Montevideo: Ediciones Trilce, 2008), 21.
} 
quienes se les plantea el reto de moverse entre la literatura, como narradores de experiencias vivenciales; el periodismo, en la práctica de la entrevista y la construcción de perfiles, y la historia como tal, de la cual no dudan en aprovechar sus métodos críticos y la producción historiográfica disponible para conectar y enmarcar los relatos de los jubilados, trascendiendo el ejercicio meramente evocativo que amenaza con condenar al pasado a ser objeto de vitrina.

La condición que hace posible la obra es el desarrollo de un ejercicio extendido de historia oral con la Asociación de Jubilados: sus integrantes participaron de talleres colectivos de memoria, entrevistas individuales y abrieron las puertas de su cotidianidad a los autores. En consecuencia, además de revelar acontecimientos, muchas veces, indetectables en la documentación de archivo, el texto consigue reconocer el entramado de significados en el cual se cimenta la experiencia histórica de quienes ocuparon los cargos directivos, docentes, administrativos y de servicios en la Universidad Tecnológica de Pereira, a lo largo de su consolidación como institución de educación superior; y, especialmente, en el contexto de agitación política y revolución cultural que caracterizó las décadas de los años sesenta, setenta y ochenta. Se aprovechó la subjetividad para presentar las voces de las personas no solo en términos de lo factual, sino de sus motivaciones, su percepción de los hechos y la manera en que hoy valoran el pasado desde una posición reflexiva ${ }^{2}$.

La estructura está organizada en diez capítulos, entre los cuales no es evidente un hilo conductor que sugiera un modo de lectura lineal. Antes bien, cada uno de ellos goza de autonomía e independencia, tanto en el estilo como en los contenidos. Por tal razón, me atrevo a presentarlos agrupados en tres secciones: del 2 al 7 contienen la narración de ejercicios de memoria colectiva que refieren al estamento de secretarias y trabajadores, así como a la Facultad de Ingeniería Eléctrica, Mecánica e Industrial; Estudios Básicos; Educación; Bellas Artes y Humanidades y Ciencias de la Salud. El segundo grupo está conformado por los capítulos 8 y 10, que dan cuenta de las memorias de exrectores y de la Asociación Sindical de Profesores Universitarios, respectivamente, coincidiendo en la discusión política propia de las visiones sobre cómo debe dirigirse la Universidad. Por último, los capítulos 1 y 9, que constituyen el tercer grupo, son relatos del presente que articulan la experiencia cotidiana de los jubilados con la memoria de su trayectoria laboral.

El proceso de investigación con fuentes orales supone un doble reto para los historiadores: de un lado, la planeación y conducción de una conversación, que para este caso cuenta con la participación de múltiples intervinientes; de otro lado, la narración de la experiencia. ¿Cómo hilar anécdotas, explicaciones y opiniones? ¿Qué lugar debe ocupar la voz de los autores? ¿Cuáles son los criterios de selección entre el amplio universo testimonial de los narradores? Sobre este tipo de interrogantes atina la obra en señalar distintos caminos que conducen a afinar las herramientas propias del historiador. Los autores optan por mantener una voz que introduce al lector con breves pero suficientes referencias al contexto nacional e internacional de la época; luego, describen la situación de la entrevista colectiva, y paulatinamente van dando entrada a las voces de los narradores con las que se van construyendo imágenes dinámicas que recrean las atmósferas de la vida universitaria y sus transformaciones a través de los años.

Las memorias de secretarias y trabajadores evocan el ambiente de camaradería que caracterizaba los primeros años de funcionamiento de la Universidad, cuando la población

\footnotetext{
${ }^{2}$ Alessandro Portelli, «Lo que hace diferente a la historia oral», en Historia Oral comp. por Dora Schwarzstein (Buenos Aires: Centro Editor de América Latina, 1991), 42.
} 
estudiantil no superaba los 2000 matriculados $^{3}$. Cabe anotar que la mirada meramente evocativa logra ser superada con la exposición de problemáticas asociadas a la consolidación de una estructura burocrática a medida que la institución crecía, presentándose además dinámicas de explotación laboral y un sinnúmero de conflictos interpersonales en los cuales fueron las mujeres las mayores perjudicadas, situación que terminó afectando las relaciones laborales. Es llamativo también el hecho de abordar el mundo de la lucha sindical desde la memoria, con lo que los autores logran desmantelar una lectura reducida a lo ideológico. Se muestra que la participación en sindicatos y movilizaciones obedecía también a afinidades personales y prácticas colectivas, descomponiendo así una visión generalista de la lucha política universitaria.

El recorrido por cada una de las facultades consigue ordenar de forma horizontal los itinerarios de todos los campos académicos, atendiendo a una de las grandes críticas a la que es susceptible la imagen oficial de una «universidad de corte ingenieril», sustentada sobre todo en el relato fundacional de los primeros programas. En el trasegar de cada cuerpo docente se pone en primer plano la acción humana como matriz explicativa de las decisiones curriculares, las trayectorias de formación profesional, las relaciones con la industria y la participación en la política universitaria. Esferas que se reconstruyen no de manera independiente, sino como un entramado de significados no ajeno a encuentros y confrontaciones. A lo anterior, se suma el reconocimiento que se hace de los legados de múltiples profesores, con lo que se rescatan historias de vida que se construyen a pulso y reclaman su espacio en la memoria institucional.

Capítulo a capítulo, los autores logran traducir las militancias políticas de la época en experiencias concretas de los actores. En tal sentido, abordan el contexto de emergencia de la «nueva izquierda», que contribuyó a la formación de una atmósfera de disputa permanente entre los militantes del Partido Comunista Colombiano (PCC) — de línea «Moscú»—, los del Movimiento Obrero Independiente y Revolucionario (MOIR) — de línea «Pekín»- y los independientes que no se reconocían en ninguna de las dos organizaciones mayoritarias, pero no por ello se excluían de participar en asambleas, protestas y contiendas electorales internas que hacían parte de la cotidianidad universitaria.

Otro aporte de suma relevancia para comprender el carácter político de la Universidad se encuentra en la reconstrucción del movimiento profesoral de 1991. La protesta se desató tras conocerse que la administración de Gabriel Jaime Cardona no había comunicado a tiempo una decisión del Consejo de Estado que anulaba algunos beneficios conseguidos por los docentes en una negociación colectiva de la Asociación Sindical de Profesores Universitarios, por lo que se vencieron los términos de apelación. Las semanas de cese de actividades y toma del campus son evocadas por los jubilados como un ambiente de verdadero colegaje, compromiso, resistencia y creatividad. Para ese entonces, los profesores consiguieron simpatizar con distintos sectores de la sociedad, lo que conllevó al nombramiento de un nuevo rector y se inició una etapa especial en la cual fueron los mismos profesores quienes asumieron la dirección de la institución.

Las rectorías de Ricardo Orozco (1991-1994), Javier Arroyave (1995-1996) y Carlos Alberto Ossa (1997-1999) son recordadas por las buenas relaciones entabladas entre los estamentos de la Universidad, después de años en que estas habían estado turbadas por las

\footnotetext{
${ }^{3} \mathrm{El}$ ambiente de buenas relaciones entre estudiantes, docentes y administrativos, característico de la etapa fundacional de la UTP, también se señala en Álvaro Acevedo, Diana Rodríguez y Nelson Giraldo, Jorge Roa Martínez. Memoria de una visión cosmopolita (Pereira: Universidad Tecnológica de Pereira, 2009), 303.
} 
diferencias que se presentaban con la administración. Se reconocen también los avances en materia de investigación y planeación estratégica, gracias a los cuales se cimentaron muchos de los ejes que han hecho posible el crecimiento y posicionamiento de la Universidad en el ámbito regional y nacional. Por otro lado, la imagen del exrector Luis Enrique Arango (20002014) suscita sensibilidades entre los jubilados, ya que muchos de ellos fueron sus compañeros en la década de los setenta cuando era reconocido como un líder sindical del MOIR. Luego se convirtieron en sus contradictores al considerar que sus iniciativas en la rectoría iban en detrimento de la visión de universidad que siempre habían defendido, por temas como la creación de programas de operación comercial, el decrecimiento de la planta docente y la postergación en el cargo de rector por cinco períodos consecutivos. Para zanjar la discusión, los autores deciden presentar la versión del ingeniero y exrector Arango como «un capítulo aparte», con lo que reiteran que el libro, lejos de querer imponer un nuevo relato contra oficial, abre espacios para que la Universidad sea comprendida a partir del disenso que le es inherente.

El libro tiene el sello de la Asociación de Jubilados, por lo que es natural que se presenten en el texto algunos apartes dedicados a la exposición de su funcionamiento y la promoción para la vinculación, los cuales generan ruido si se pretende una lectura netamente académica. Sin embargo, aunque podría considerarse que el público al que se encuentra dirigido son los jubilados que no hacen parte aún de la Asociación, es de resaltar que el trabajo de los autores ha sido juicioso al incorporar los elementos propios del discurso oficial de la AJUTP. Hay una reflexión general sobre la memoria como una actividad que siempre parte del presente, por lo que los capítulos 1 y 9 aportan a la comprensión de un estamento que se integra de forma activa con la comunidad universitaria.

Por último, invito a reflexionar en el sentido que propone Portelli, cuando menciona que «la historia oral nunca puede contarse sin tomar partido, ya que los partidos existen en

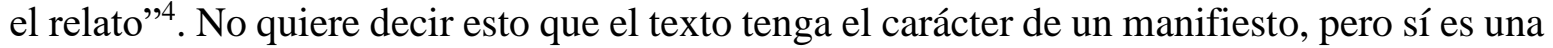
voz - construida a su vez de múltiples voces - que invita a pensar en el retroceso que se ha dado en materia laboral en los últimos años, sospecha de los relatos oficiales que desconocen los legados de trayectorias personales, reivindica el carácter político de la Universidad y convida a los historiadores a revisar las historias institucionales a la luz de las experiencias que aportan las fuentes orales. Ello no implica desconocer la riqueza de otro tipo de fuentes, sino asignarles un lugar de predominancia que permita no solo exponer una versión del pasado. Fundamentalmente, dotarlo de sentido para la reflexión sobre el presente en la perspectiva de ofrecer relatos incluyentes, que propongan nuevos interrogantes y motiven la discusión al interior de las comunidades universitarias.

\section{Referencias}

Acevedo, Álvaro, Diana Rodríguez y Nelson Giraldo. Jorge Roa Martínez. Memoria de una visión cosmopolita. Pereira: Universidad Tecnológica de Pereira, 2009.

Pierre, Nora. Los lugares de la memoria. Montevideo: Ediciones Trilce, 2008.

\footnotetext{
${ }^{4}$ Portelli, «Lo que hace diferente a la historia oral», 51.
} 
Portelli, Alessandro. «Lo que hace diferente a la historia oral». En Historia Oral, compilado por Dora Schwarzstein, 42. Buenos Aires: Centro Editor de América Latina, 1991 